\title{
Numerical Solution of Nonlinear Fredholm Integro-Differential Equations Using Spectral Homotopy Analysis Method
}

\author{
Z. Pashazadeh Atabakan, A. Kazemi Nasab, A. Kılıçman, and Zainidin K. Eshkuvatov \\ Department of Mathematics, University Putra Malaysia (UPM), 43400 Serdang, Selangor, Malaysia \\ Correspondence should be addressed to A. Kilıçman; akilicman@putra.upm.edu.my
}

Received 25 February 2013; Revised 18 April 2013; Accepted 20 April 2013

Academic Editor: Fazal M. Mahomed

Copyright (c) 2013 Z. Pashazadeh Atabakan et al. This is an open access article distributed under the Creative Commons Attribution License, which permits unrestricted use, distribution, and reproduction in any medium, provided the original work is properly cited.

\begin{abstract}
Spectral homotopy analysis method (SHAM) as a modification of homotopy analysis method (HAM) is applied to obtain solution of high-order nonlinear Fredholm integro-differential problems. The existence and uniqueness of the solution and convergence of the proposed method are proved. Some examples are given to approve the efficiency and the accuracy of the proposed method. The SHAM results show that the proposed approach is quite reasonable when compared to homotopy analysis method, Lagrange interpolation solutions, and exact solutions.
\end{abstract}

\section{Introduction}

The integro-differential equations stem from the mathematical modeling of many complex real-life problems. Many scientific phenomena have been formulated using integrodifferential equations $[1,2]$. Solving nonlinear integrodifferential equation is much more difficult than linear one analytically. So different types of numerical methods have been used to obtain an efficient approximation solution $[3,4]$. In 1992 Liao [5] proposed the homotopy analysis method (HAM) concept in topology for solving nonlinear differential equations. Liao $[6,7]$ found that the convergence of series solutions of nonlinear equations cannot be guaranteed by the early HAM. Further, Liao [6] introduced a nonzero auxiliary parameter to solve this limitation. Unlike the special cases of HAM such as Lyapunove's artificial small parameter method [8], Adomian decomposition method [9-12], and the $\delta$ expansion method [13], this method need not a small perturbation parameter. In the HAM the perturbation techniques [14] need not be converted a nonlinear problem to infinite number of linear problems. The homotopy analysis method is applicable for solving problems having strong nonlinearity [15], even if they do not have any small or large parameters, so it is more powerful than traditional perturbation methods.

The convergence region and the rate of approximation in series can been adjusted by this method. Also it can give us freedom to use different base function to approximate a non linear problem. The convergence of HAM for solving Volterra-Fredholm integro-differential equations is presented in [16].

In 2010, Motsa et al. [17] suggested the so-called spectral homotopy analysis method (SHAM) using the Chebyshev pseudospectral method to solve the linear high-order deformation equations. Since the SHAM combines the HAM with the numerical techniques, it provides us larger freedom to choose auxiliary linear operators. Thus, one can choose more complicated auxiliary linear operators in the frame of the SHAM. In theory, any continuous function in a bounded interval can be best approximated using Chebyshev polynomial. So, the SHAM provides larger freedom to choose the auxiliary linear operator $L$ and initial guess. Further, it is easy to employ the optimal convergence-control parameter in the frame of the SHAM. Thus, the SHAM has great potential to solve more complicated nonlinear problems in science and engineering, although further modifications in theory and more applications are needed. Chebyshev polynomial is considered a kind of special function. There are many other special functions such as Hermite polynomial, Legendre polynomial, Airy function, Bessel function, Riemann zeta function, and hypergeometric functions. Since the HAM provides us extremely large freedom to choose the auxiliary linear operator $L$ and the initial guess, it should be possible 
to develop a generalized spectral HAM which can use a proper special function for a given nonlinear problem. The spectral homotopy analysis method has been used for solving partial and ordinary differential equations [18-20]. Spectral homotopy analysis method and its convergency for solving a class of optimal control problems are presented in [21]. Motsa et al. [17-19] found that the spectral homotopy analysis method is more efficient than the homotopy analysis method as it does not depend on the rule of solution expression and the rule of ergodicity. This method is more flexible than homotopy analysis method, since it allows for a wider range of linear and nonlinear operators, and one is not restricted to use the method of higher-order differential mapping for solving boundary value problems in bounded domains, unlike the homotopy analysis method. The range of admissible $h$ values is much wider in spectral homotopy analysis method than in homotopy analysis method. The main restriction of HAM in solving integral equations is to choose the best initial guess, as the series solution is convergent. In SHAM the initial approximation is taken to be the solution of the nonhomogeneous linear part of the given equation. In 2012, Pashazadeh Atabakan et al. solved linear Volterra and Fredholm integro-differential equations using spectral homotopy analysis method; see [22].

In this paper, we apply spectral homotopy analysis method (SHAM) to solve higher-order nonlinear Fredholm type of integro-differential equations. Fredholm integrodifferential equation is given by

$$
\begin{gathered}
\sum_{j=0}^{2} a_{j}(x) y^{(j)}(x)=f(x)+\mu \int_{-1}^{1} k(x, t)[y(t)]^{r} d t \\
y(-1)=y(1)=0,
\end{gathered}
$$

where $\mu$ is constant value, $f(x), k(x, t),[y(t)]^{r}$, and $a_{j}(x) r \geq 1$ are functions that have suitable derivatives on interval $-1 \leq$ $t \leq x \leq 1$ and $a_{2}(x) \neq 0$.

The paper is organized in the following way. Section 2 includes a brief introduction in homotopy analysis method. Spectral homotopy analysis method for solving nonlinear Fredholm integral equations is presented in Section 3. The existence and uniqueness of the solution and convergence of the proposed method are proved in Section 4. In Section 5, numerical examples are presented. In Section 6, concluding remarks are given.

\section{Homotopy Analysis Solution}

In this section, we give a brief introduction to HAM. We consider the following differential equation in a general form as follows:

$$
N[y(\eta)]=0,
$$

where $N$ is nonlinear operator, $\eta$ denotes independent variables, and $y(\eta)$ is an unknown function, respectively. For simplicity we disregard all initial and all boundary conditions which can be dealt in similar way. The so-called zero-order deformation equation was constructed by Liao as follows:

$$
(1-p) L\left[\psi(\eta ; p)-y_{0}(\eta)\right]=p h H(\eta)(N[\psi(\eta ; p)]),
$$

where $p \in[0,1]$ is the embedding parameter, $h$ is a nonzero convergence-parameter, $H(\eta)$ is an auxiliary function, $y_{0}(\eta)$ is called an initial guess of $y(\eta)$, and $\psi(\eta ; p)$ is an unknown function. In addition, $L$ is an auxiliary linear operator, and $N$ is nonlinear operator as follows:

$$
L(\psi(x ; p))=a_{k}(x) \frac{\partial^{2} \psi(x ; p)}{\partial x^{2}}
$$

with the property $L\left(\sum_{j=0}^{2} c_{j} t^{j}\right)=0$, where $c_{j}$, are constants and

$$
\begin{aligned}
N[\psi(x ; p)]= & \sum_{j=0}^{2} a_{j}(x) \frac{\partial^{j} \psi(x ; p)}{\partial x^{j}}-f(x) \\
& -\mu \int_{-1}^{1} k(x, t) \psi^{r}(t) d t
\end{aligned}
$$

is a nonlinear operator. Obviously, when $p=0$ and $p=1$, it holds $\psi(\eta ; 0)=y_{0}(\eta)$ and $\psi(\eta ; 1)=y(\eta)$. In this way, as $p$ increase from 0 to $1, \psi(\eta ; p)$ alter from initial guess $y_{0}(\eta)$ to the solution $y(\eta)$, and $\psi(\eta ; p)$ is expanded in Taylor series with respect to $p$ as follows:

$$
\psi(\eta ; p)=y_{0}(\eta)+\sum_{m=1}^{+\infty} y_{m}(\eta) p^{m}
$$

where

$$
\begin{aligned}
y_{m}(\eta) & =D_{m}[\psi(\eta ; p)], \\
D_{m} \psi & =\left.\frac{1}{m !} \frac{\partial^{m} \psi}{\partial p^{m}}\right|_{p=0} .
\end{aligned}
$$

The series (6) converges at $p=1$ if the auxiliary linear operator, the initial guess, the convergence-parameter, and the auxiliary function are properly selected as follows:

$$
\psi(\eta)=y_{0}(\eta)+\sum_{m=1}^{+\infty} y_{m}(\eta) .
$$

The admissible and valid values of the convergenceparameter $h$ are found from the horizontal portion of the $h$-curves. Liao proved that $y(\eta)$ is one of the solutions of original nonlinear equation. As $H(\eta)=1$, so (3) becomes

$$
(1-p) L\left[\psi(\eta ; p)-y_{0}(\eta)\right]=\operatorname{ph}(N[\psi(\eta ; p)]) .
$$

Define the vector $y_{m}=\left\{y_{0}(\eta), y_{1}(\eta), \ldots, y_{m}(\eta)\right\}$. Operating on both side of (9) with $D_{m}$, we have the so called $m$ th-order deformation equation as follows:

$$
L\left[y_{m}(\eta)-\chi_{m} y_{m-1}(\eta)\right]=h H(\eta) R_{m}\left(y_{m-1}(\eta)\right),
$$


where

$$
\begin{gathered}
R_{m}\left(y_{m-1}\right)=\left.\frac{1}{(m-1) !} \frac{\partial^{m-1} N[\psi(\eta ; p)]}{\partial p^{m-1}}\right|_{p=0}, \\
\chi_{m}= \begin{cases}0, & m \leq 1 \\
1, & \text { otherwise }\end{cases}
\end{gathered}
$$

$y_{m}(\eta)$ for $m \geq 0$ that is governed by the linear equation (10) can be solved by symbolic computation software such as MAPLE, MATLAB, and similar CAS.

\section{Spectral-Homotopy Analysis Solution}

Consider the non linear Fredholm integro-differential equation:

$$
\begin{gathered}
\sum_{j=0}^{2} a_{j}(x) y^{(j)}(x)=f(x)+\mu \int_{-1}^{1} k(x, t)[y(t)]^{r} d t, \\
y(-1)=y(1)=0 .
\end{gathered}
$$

We begin by defining the following linear operator:

$$
L(\psi(x ; p))=\sum_{j=0}^{2} a_{j}(x) \frac{\partial^{j} \psi(x ; p)}{\partial x^{j}},
$$

where $p \in[0,1]$ is the embedding parameter and $\psi(x ; p)$ is an unknown function. The zeroth-order deformation equation is given by

$$
(1-p) L\left[\psi(\eta ; p)-y_{0}(\eta)\right]=p h(N[\psi(\eta ; p)]-f(\eta)),
$$

where $h$ is the nonzero convergence controlling auxiliary parameter and $N$ is a nonlinear operator given by

$$
\begin{aligned}
N[\psi(\eta ; p)]= & \sum_{j=0}^{2} a_{j}(\eta) \frac{\partial^{j} \psi(\eta ; p)}{\partial \eta^{j}}-f(\eta) \\
& -\mu \int_{-1}^{1} k(\eta, t) \psi^{r}(t) d t .
\end{aligned}
$$

Differentiating (14) $m$ times with respect to the embedding parameter $p$, setting $p=0$, and finally dividing them by $m$ !, we have the so called $m$ th-order deformation equation

$$
L\left[y_{m}(\eta)-\chi_{m} y_{m-1}(\eta)\right]=h R_{m},
$$

subject to boundary conditions

$$
y_{m}(-1)=y_{m}(1)=0,
$$

where

$$
\begin{aligned}
R_{m}(\eta)= & \sum_{j=0}^{2} a_{j}(\eta) \frac{\partial^{j} \psi(\eta ; p)}{\partial \eta^{j}}-f(\eta)\left(1-\chi_{m}\right) \\
& -\mu \int_{-1}^{1} k(\eta, t) \psi^{r}(t) d t .
\end{aligned}
$$

The initial approximation that is used in the higher-order equation (18) is obtained on solving the following equation:

$$
\sum_{j=0}^{2} a_{j}(x) y_{0}^{(j)}(x)=f(x)
$$

subject to boundary conditions

$$
y_{0}(-1)=y_{0}(1)=0 \text {, }
$$

where we use the Chebyshev pseudospectral method to solve (19)-(20).

We first approximate $y_{0}(\eta)$ by a truncated series of Chebyshev polynomial of the following form:

$$
y_{0}(\eta) \approx y_{0}^{N}\left(\eta_{j}\right)=\sum_{k=0}^{N} \widehat{y}_{k} T_{k}\left(\eta_{j}\right), \quad j=0, \ldots, N,
$$

where $T_{k}$ is the $k$ th Chebyshev polynomials, $\widehat{y}_{k}$ are coefficients and Gauss-Lobatto collocation points $\eta_{0}, \eta_{1}, \ldots, \eta_{N}$ which are the extrema of the Nth-order Chebyshev polynomial defined by

$$
\eta_{j}=\cos \left(\frac{\pi j}{N}\right)
$$

Derivatives of the functions $y_{0}(\eta)$ at the collocation points are represented as

$$
\frac{d^{s} y_{0}\left(\eta_{k}\right)}{d \eta^{s}}=\sum_{j=0}^{N} D_{k j}^{s} y_{0}\left(\eta_{j}\right), \quad k=0, \ldots, N
$$

where $s$ is the order of differentiation and $D$ is the Chebyshev spectral differentiation matrix. Following [23], we express the entries of the differentiation matrix $D$ as

$$
\begin{gathered}
D_{k j}=\left(\frac{-1}{2}\right) \frac{c_{k}}{c_{j}} \\
\quad \times \frac{(-1)^{k+j}}{\sin (\pi(j+k) / 2 N) \sin (\pi(j-k) / 2 N)}, \quad j \neq k, \\
D_{k j}=\left(\frac{-1}{2}\right) \frac{x_{k}}{\sin ^{2}(\pi k / N)}, \quad k \neq 0, N, k=j, \\
D_{00}=-D_{N N}=\frac{2 N^{2}+1}{6} .
\end{gathered}
$$

Substituting (21)-(23) into (19) will result in

$$
\mathrm{AY}_{0}=\mathbf{F}
$$

subject to the boundary conditions

$$
y_{0}\left(\eta_{0}\right)=y_{0}\left(\eta_{N}\right)=0 \text {, }
$$


where

$$
\begin{gathered}
\mathbf{A}=\sum_{j=0}^{2} \mathbf{a}_{j} \mathbf{D}^{j}, \\
\mathbf{Y}_{0}=\left[y_{0}\left(\eta_{0}\right), y_{0}\left(\eta_{1}\right), \ldots, y_{0}\left(\eta_{N}\right)\right]^{T}, \\
\mathbf{F}=\left[f\left(\eta_{0}\right), f\left(\eta_{1}\right), \ldots, f\left(\eta_{N}\right)\right]^{T}, \\
\mathbf{a}_{r}=\operatorname{diag}\left(a_{r}\left(\eta_{0}\right), a_{r}\left(\eta_{1}\right), \ldots, a_{r}\left(\eta_{N}\right)\right) .
\end{gathered}
$$

The values of $y_{0}\left(\eta_{i}\right), i=0, \ldots, N$ are determined from the equation

$$
\mathbf{Y}_{0}=\mathbf{A}^{-1} \mathbf{F},
$$

which is the initial approximation for the SHAM solution of the governing equation (12). Apply the Chebyshev pseudospectral transformation on (16)-(18) to obtain the following result:

$$
\mathbf{A Y}_{m}=\left(\chi_{m}+h\right) \mathbf{A Y}_{m-1}-h\left[\mathbf{S}_{m-1}-\left(1-\chi_{m}\right) \mathbf{F}\right],
$$

subject to the boundary conditions

$$
y_{m}\left(\eta_{0}\right)=y_{m}\left(\eta_{N}\right)=0,
$$

where $\mathbf{A}$ and $\mathbf{F}$ were defined in and

$$
\begin{gathered}
\mathbf{Y}_{m}=\left[y_{m}\left(\eta_{0}\right), y_{m}\left(\eta_{1}\right), \ldots, y_{m}\left(\eta_{N}\right)\right]^{T}, \\
\mathbf{s}_{m}=\int_{-1}^{1} k(\tau, t)\left[\mathbf{Y}_{m}\right]^{r} d t .
\end{gathered}
$$

To implement the boundary condition (30) we delete the first and the last rows of $\mathbf{S}_{m-1}, \mathbf{F}$ and the first and the last rows and columns of A. Finally this recursive formula can be written as follows:

$$
\mathbf{Y}_{m}=\left(\chi_{m}+h\right) \mathbf{Y}_{m-1}-h \mathbf{A}^{-1}\left[\mathbf{S}_{m-1}-\left(1-\chi_{m}\right) \mathbf{F}_{m-1}\right],
$$

with starting from the initial approximation we can obtain higher-order approximation $\mathbf{Y}_{m}$ for $m \geq 1$ recursively. To compute the integral in (32) we use the Clenshaw-Curtis quadrature formula as follows:

$$
\mathbf{S}_{m}(\eta)=\int_{-1}^{1} k\left(\eta, t, \widetilde{\mathbf{Y}}_{m}\right) d t=\sum_{j=0}^{N} w_{j} k\left(\eta, \eta_{j}, \widetilde{\mathbf{Y}}_{m}\right),
$$

where the nodes $\eta_{j}$ are given by (22) and the weights $w_{j}$ are given by

$$
\begin{gathered}
w_{0}=w_{N}= \begin{cases}\frac{1}{N^{2}}, & N \text { odd, } \\
\frac{1}{N^{2}-1}, & N \text { even, }\end{cases} \\
w_{l}=\frac{2}{N \gamma_{l}}\left[1-\sum_{k=1}^{\lfloor N / 2\rfloor} \frac{2}{\gamma_{2 k}\left(4 k^{2}-1\right)} \cos \frac{2 k l \pi}{N}\right], \\
l=1, \ldots, N-1,
\end{gathered}
$$

where $\gamma_{0}=\gamma_{N}=2$ and $\gamma_{l}=1$, for $l=1, \ldots, N-1$. $\widetilde{\mathbf{Y}}$ is a column vector of the elements of the vector $\mathbf{Y}$ that is computed as follows:

$$
\widetilde{\mathbf{Y}}_{m}=\sum_{n_{1}=0}^{m} y_{m-n_{1}} \sum_{n_{2}=0}^{n_{1}} y_{n_{1}-n_{2}} \cdots \sum_{n_{r-1}=0}^{n_{r-2}} y_{n_{r-2}-n_{r-1}} y_{n_{r-1}},
$$

where $m, r \geq 0$ are positive integers [24].

Regarding to accuracy, the stability, and the error of previous quadrature formula at the Gauss-Lobatto points we refer the reader to [25].

\section{Convergence Analysis}

Following the authors in $[7,16,26]$, we present the convergence of spectral homotopy analysis method for solving Fredholm integro-differential equations.

In view of (13) and (27), (12) can be written as follows:

$$
\mathbf{A Y}=\mathbf{F}+\mu \int_{-1}^{1} k(x, t) \mathbf{G}(\mathbf{Y}) d t
$$

where $\mathbf{Y}, \mathbf{F}$, and $\mathbf{G}(\mathbf{Y})$ are vector functions.

We obtain

$$
\mathbf{Y}=\mathbf{A}^{-1} \mathbf{F}+\mu \int_{-1}^{1} k(x, t) \mathbf{A}^{-1} \mathbf{G}(\mathbf{Y}) d t
$$

By substituting $\widetilde{\mathbf{F}}=\mathbf{A}^{-1} \mathbf{F}$ and $\widetilde{\mathbf{G}}(\mathbf{Y})=\mathbf{A}^{-1} \mathbf{G}(\mathbf{Y})$ in (38), we obtain

$$
\mathbf{Y}=\widetilde{\mathbf{F}}+\mu \int_{-1}^{1} k(x, t) \widetilde{\mathbf{G}}(\mathbf{Y}) d t .
$$

In (39), we assume that $\widetilde{\mathbf{F}}$ is bounded for all $t$ in $C=[-1,1]$ and

$$
|k(x, t)| \leq M .
$$

Also, we suppose that the non linear term $\widetilde{\mathbf{G}}(\mathbf{Y})$ is Lipschitz continuous with

$$
\left\|\widetilde{\mathbf{G}}(\mathbf{Y})-\widetilde{\mathbf{G}}\left(\mathbf{Y}^{*}\right)\right\| \leq L\left\|\mathbf{Y}-\mathbf{Y}^{*}\right\| .
$$

If we set $\alpha=2 \mu L M$, then the following can be proved by using the previous assumptions.

Theorem 1. The nonlinear Fredholm integro-differential equation in (32) has a unique solution whenever $0<\alpha<1$.

Proof. Let $\mathbf{Y}$ and $\mathbf{Y}^{*}$ be two different solutions of (39), then

$$
\begin{aligned}
\left\|\mathbf{Y}-\mathbf{Y}^{*}\right\| & =\left\|\mu \int_{-1}^{1} k(x, t)\left[\widetilde{\mathbf{G}}(\mathbf{Y})-\widetilde{\mathbf{G}}\left(\mathbf{Y}^{*}\right)\right] d t\right\| \\
& \leq \mu \int_{-1}^{1}|k(x, t)|\left\|\widetilde{\mathbf{G}}(\mathbf{Y})-\widetilde{\mathbf{G}}\left(\mathbf{Y}^{*}\right)\right\| d t \\
& \leq 2 \mu L M\left\|\mathbf{Y}-\mathbf{Y}^{*}\right\| .
\end{aligned}
$$

So we get $(1-\alpha)\left\|\mathbf{Y}-\mathbf{Y}^{*}\right\| \leq 0$. Since $0<\alpha<1$, so $\left\|\mathbf{Y}-\mathbf{Y}^{*}\right\|=0$, therefore $\mathbf{Y}=\mathbf{Y}^{*}$, and this completes the proof. 


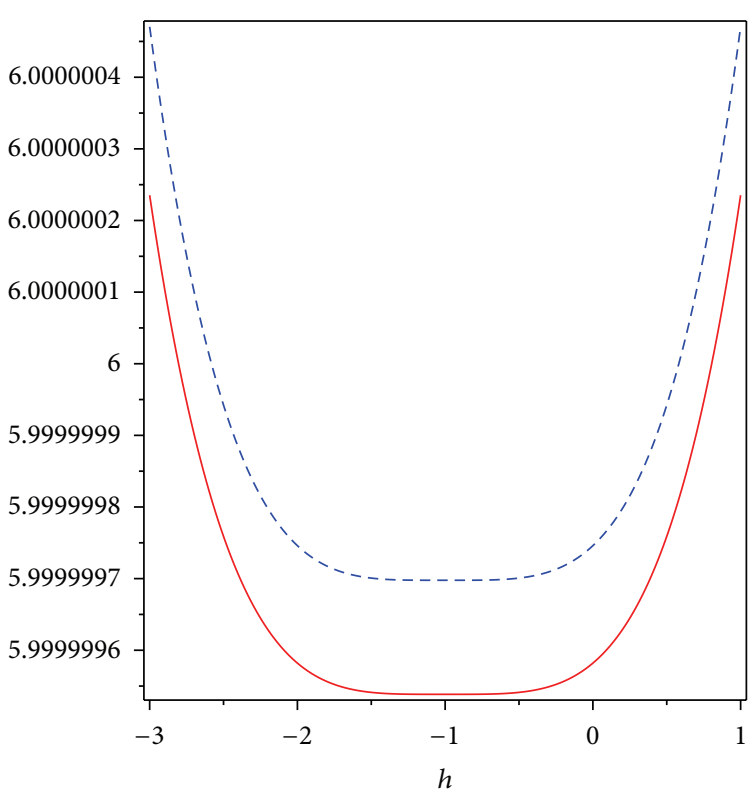

(a)

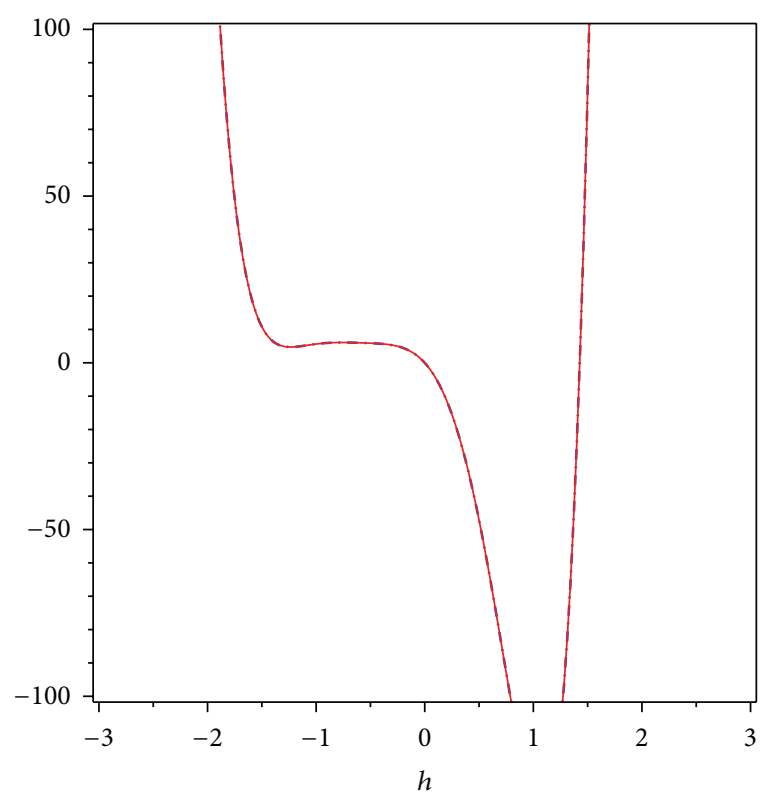

(b)

FIgURE 1: The $h$-curve $y^{\prime \prime}(0)$ and $y^{\prime \prime \prime}(0)$ for 10th-order (a) SHAM, (b) HAM.

Theorem 2. If the series solution $\mathbf{Y}=\sum_{m=0}^{\infty} \mathbf{Y}_{m}$ obtained from (32) is convergent, then it converges to the exact solution of the problem (39).

Proof. We assume

$$
\mathbf{Y}=\sum_{m=0}^{\infty} \mathbf{Y}_{m}, \quad \mathbf{V}(\mathbf{Y})=\sum_{m=0}^{\infty} \widetilde{\mathbf{G}}\left(\mathbf{Y}_{m}\right)
$$

where $\lim _{m \rightarrow \infty} \mathbf{Y}_{m}=0$. We can write

$$
\begin{aligned}
\sum_{m=1}^{n}\left[\mathbf{Y}_{m}-\chi_{m} \mathbf{Y}_{m-1}\right] \\
\quad=\mathbf{Y}_{1}+\left(\mathbf{Y}_{2}-\mathbf{Y}_{1}\right)+\cdots+\left(\mathbf{Y}_{n}-\mathbf{Y}_{n-1}\right)=\mathbf{Y}_{n} .
\end{aligned}
$$

Hence, from (44),

$$
\sum_{m=1}^{\infty}\left[\mathbf{Y}_{m}-\chi_{m} \mathbf{Y}_{m-1}\right]=0,
$$

so using (45) and the definition of the linear operator $L$, we have

$$
\sum_{m=1}^{\infty} L\left[\mathbf{Y}_{m}-\chi_{m} \mathbf{Y}_{m-1}\right]=L\left[\sum_{m=1}^{\infty} \mathbf{Y}_{m}-\chi_{m} \mathbf{Y}_{m-1}\right]=0 .
$$

Therefore, from (16), we can obtain that

$$
\sum_{m=1}^{\infty} L\left[\mathbf{Y}_{m}-\chi_{m} \mathbf{Y}_{m-1}\right]=h \sum_{m=1}^{\infty} R_{m}\left(\mathbf{Y}_{m-1}\right)=0 .
$$

Since $h \neq 0$, we have

$$
\sum_{m=1}^{\infty} R_{m}\left(\mathbf{Y}_{m-1}\right)=0
$$

By applying (39) and (43),

$$
\begin{aligned}
& \sum_{m=1}^{\infty} R_{m}\left(\mathbf{Y}_{m-1}\right) \\
& \quad=\sum_{m=1}^{\infty}\left[\mathbf{Y}_{m-1}-\left(1-\chi_{m-1}\right) \widetilde{\mathbf{F}}-\mu \int_{-1}^{1} k(x, t) \widetilde{\mathbf{G}}\left(\mathbf{Y}_{m-1}\right) d t\right] \\
& \quad=\mathbf{Y}-\widetilde{\mathbf{F}}-\mu \int_{-1}^{1} k(x, t) \mathbf{V}(\mathbf{Y}) d t .
\end{aligned}
$$

Therefore, Y must be the exact solution of (39).

\section{Numerical Examples}

In this section we apply the technique described in Section 3 to some illustrative examples of higher-order nonlinear Fredholm integro-differential equations.

Example 1. Consider the second-order Fredholm integrodifferential equation

$$
y^{\prime \prime}(x)=6 x+\int_{-1}^{1} x t\left(y^{\prime}(t)\right)^{2}(y(t))^{2} d t
$$

subject to $y(-1)=y(1)=0$ with the exact solution $y(x)=$ $x^{3}-x$. We employ SHAM and HAM to solve this example. From the $h$-curves (Figure 1), it is found that when $-1.5 \leq$ $h \leq 1.5$ and $-1 \leq h \leq 0$, the SHAM solution and HAM solution converge to the exact solution, respectively. A numerical results of Example 1 against different order of SHAM approximate solutions is shown in Table 1. 
TABLE 1: The numerical results of Example 1 against different order of SHAM approximate solutions with $h=-0.01$.

\begin{tabular}{lccc}
\hline$x$ & & SHAM & Numerical \\
\hline 1.00000 & 2nd order & 4th order & 0 \\
0.99965 & 0 & 0 & -0.01162119 \\
0.99861 & -0.01162119 & -0.01162119 & -0.04513187 \\
0.99687 & -0.04513180 & -0.04513187 & -0.16001177 \\
0.99443 & -0.16001177 & -0.16001177 & -0.22774902 \\
0.99130 & -0.22774902 & -0.22774902 & -0.29155781 \\
0.98748 & -0.29155781 & -0.29155781 & -0.34334545 \\
0.98297 & -0.34334545 & -0.34334545 & -0.3760608 \\
0.97778 & -0.37606083 & -0.37606087 & -0.38445192 \\
0.97191 & -0.38445192 & -0.38445192 & -0.36563661 \\
\hline
\end{tabular}

Example 2. Consider the second order Fredholm integrodifferential equation

$$
\begin{aligned}
& x y^{\prime \prime}(x)+x^{2} y^{\prime}(x)+2 y(x) \\
& =\left(-\pi^{2} x+2\right) \sin (\pi x)+\pi x^{2} \cos (\pi x) \\
& \quad+\int_{-1}^{1} \cos (\pi t) y^{4}(t) d t
\end{aligned}
$$

subject to $y(-1)=y(1)=0$ with the exact solution $y(x)=$ $\sin (\pi x)$. We employ HAM and SHAM to solve this example. The numerical results of Example 2 against different order of SHAM approximate solutions with $h=-0.01$ is shown in Table 2. In Table 3, there is a comparison of the numerical result against the HAM and SHAM approximation solutions at different orders with $h=-0.001$. It is worth noting that the SHAM results become very highly accurate only with a few iterations, and fifth-order solutions are very close to the exact solution. Comparison of the numerical solution with the 4 thorder SHAM solution for $h=-0.01$ is made in Figure 2. As it is shown in Figure 3, the rate of convergency in SHAM is faster than HAM. In Figure 4, it is found that when $-2.5 \leq$ $h \leq 0.5$ and $-1 \leq h \leq 1$, the SHAM solution and HAM solution converge to the exact solution, respectively. In HAM we choose $y_{0}(x)=1-x^{2}$ as initial guess.

Example 3. Consider the first-order Fredholm integrodifferential equation $[27,28]$

$$
y^{\prime}(x)=-\frac{1}{2} e^{x+2}+\frac{3}{2} e^{x}+\int_{0}^{1} e^{x-t} y^{3}(t) d t
$$

subject to the boundary condition $y(0)=1$. In order to apply the SHAM for solving the given problem, we should transform using an appropriate change of variables as

$$
x=\frac{\zeta+1}{2}, \quad \zeta \in[-1,1] .
$$

Then, we use the following transformation:

$$
y(x)=Y(\zeta)+e^{(x+1) / 2} .
$$

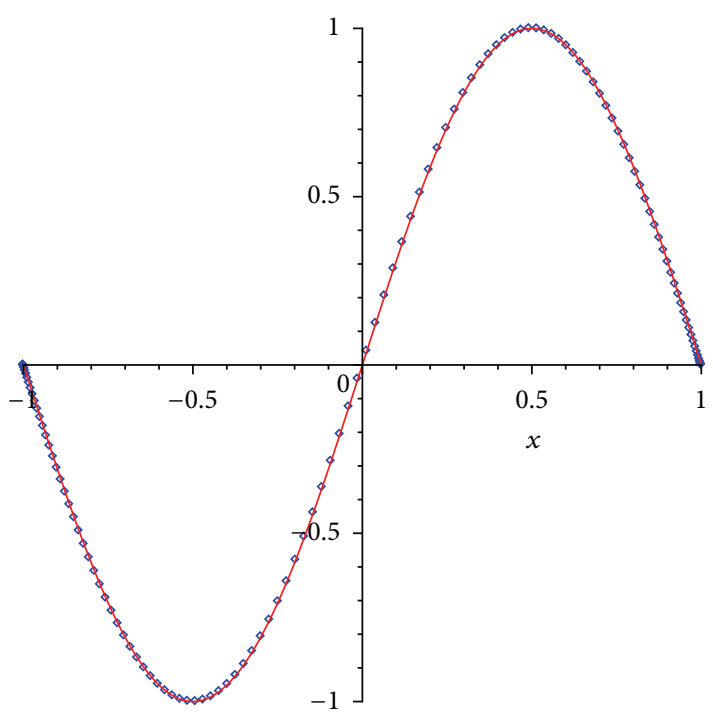

Figure 2: Comparison of the numerical solution of Example 2 with the 4 th-order SHAM solution for $h=-0.01$.

We make the governing boundary condition homogeneous. Substituting (54) into the governing equation and boundary condition results in

$$
Y^{\prime}(\zeta)=\frac{1}{4} \int_{-1}^{1} e^{(\zeta-t) / 2}\left(Y^{3}(t)+3 e^{t+1} Y(t)+3 e^{(t+1) / 2} Y^{2}(t)\right) d t
$$

subject to the boundary condition $Y(-1)=0$. A comparison between absolute errors in solutions by SHAM, Lagrange interpolation, and Rationalized Haar functions is tabulated in Table 4. It is also worth noting that the SHAM results are very close to exact solutions only with two iterations.

\section{Conclusion}

In this paper, we presented the application of spectral homotopy analysis method (SHAM) for solving nonlinear Fredholm integro-differential equations. A comparison was made between exact analytical solutions and numerical 
TABLE 2: The numerical results of Example 2 against different order of SHAM approximate solutions with $h=-0.01$.

\begin{tabular}{lcccc}
\hline$x$ & 2nd order & 3rd order & 4th order & Numerical \\
\hline 1.00000 & 0 & 0 & 0 & 0 \\
0.99965 & 0.00437807 & 0.00437807 & 0.00437807 & 0.00437807 \\
0.99861 & 0.00109471 & 0.00109471 & 0.00109471 & 0.00109471 \\
0.99687 & 0.00984768 & 0.00984768 & 0.00984768 & 0.00984768 \\
0.99443 & 0.01749926 & 0.01749926 & 0.01749926 & 0.01749926 \\
0.99130 & 0.02732631 & 0.02732631 & 0.02732631 & 0.02732631 \\
0.98748 & 0.03931949 & 0.03931949 & 0.03931950 & 0.03931950 \\
0.98297 & 0.05346606 & 0.05346607 & 0.05346607 & 0.06974900 \\
0.97778 & 0.06974898 & 0.06974899 & 0.06974899 & 0.06974900 \\
0.97191 & 0.0881459 & 0.08814599 & 0.08814599 & 0.08814600 \\
\hline
\end{tabular}

TABLE 3: Numerical result of Example 2 against the HAM and the SHAM solutions with $h=-0.001$.

\begin{tabular}{lcccccc}
\hline$x$ & & SHAM & & HAM & Numerical \\
& 5th order & 6th order & 7th order & 3rd order & 4th order & -0.0881460 \\
-0.97191 & -0.0881460 & -0.0881460 & -0.0881460 & -0.05395836 & -0.05794467 & -0.06974902 \\
-0.97778 & -0.06974902 & -0.06974902 & -0.06974902 & -0.04280765 & -0.04597139 & -0.05346607 \\
-0.98297 & -0.05346609 & -0.05346609 & -0.05346609 & -0.03289259 & -0.03532441 & -0.03931950 \\
-0.98748 & -0.03931951 & -0.03931951 & -0.03931951 & -0.02424140 & -0.02603420 & -0.02732630 \\
-0.99130 & -0.02732631 & -0.02732631 & -0.02732631 & -0.01687877 & -0.01812740 & -0.01749926 \\
-0.99443 & 0.01749926 & 0.01749926 & 0.01749926 & -0.00609972 & -0.01162680 & -0.01162680 \\
-0.99687 & -0.00984768 & -0.00984768 & -0.00984768 & -0.00609972 & -0.00984768 \\
-0.99861 & -0.00437807 & -0.00437807 & -0.00437807 & -0.00271424 & -0.00655115 & -0.00437807 \\
-0.99965 & -0.00109471 & 0.00109471 & -0.00109471 & -0.00067905 & -0.00072931 & -0.00109471 \\
-1.00000 & 0 & 0 & 0 & 0 & 0 & 0 \\
\hline
\end{tabular}

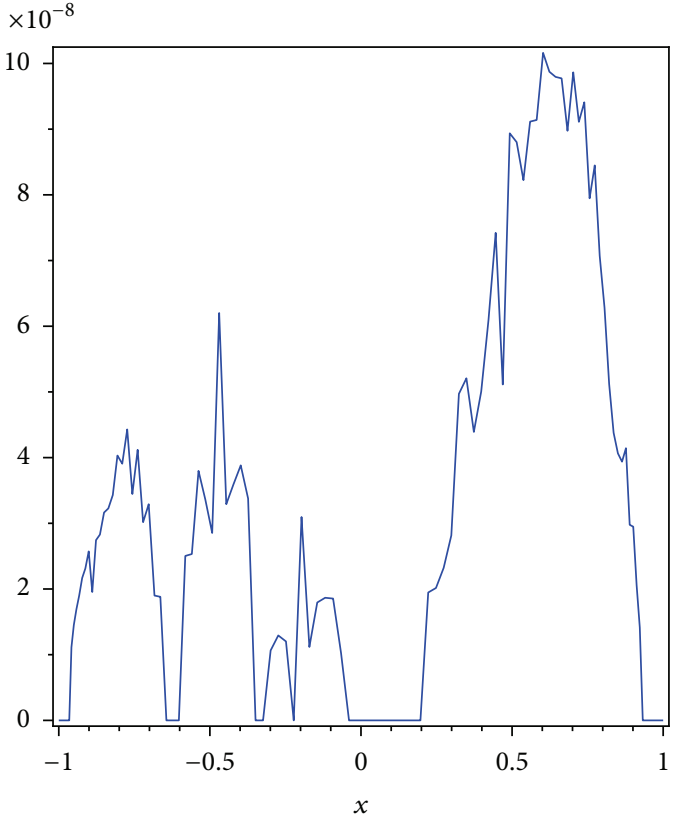

(a)

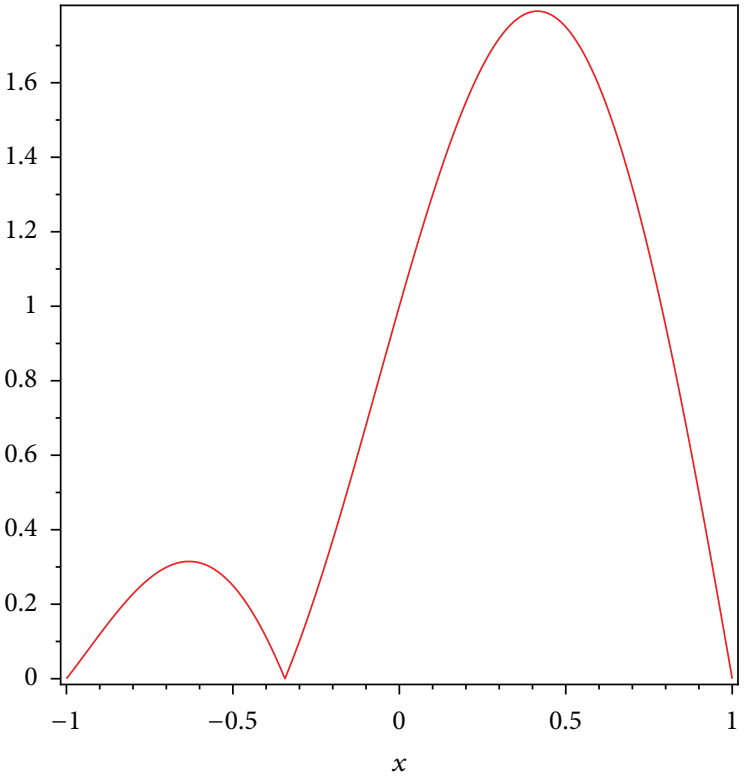

(b)

FIGURE 3: Comparison of the absolute error of third-order (a) SHAM, (b) HAM. 


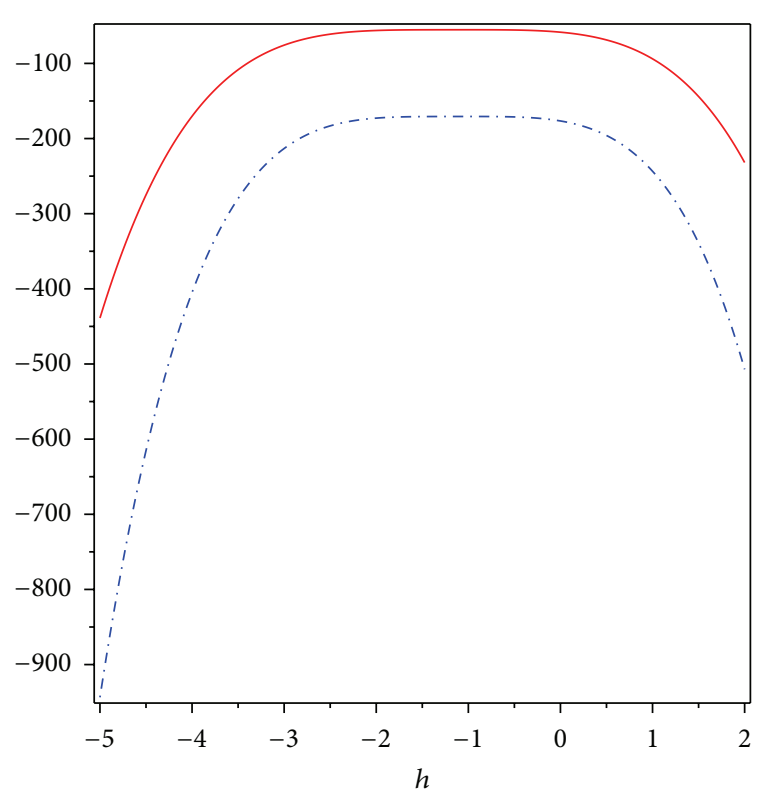

(a)

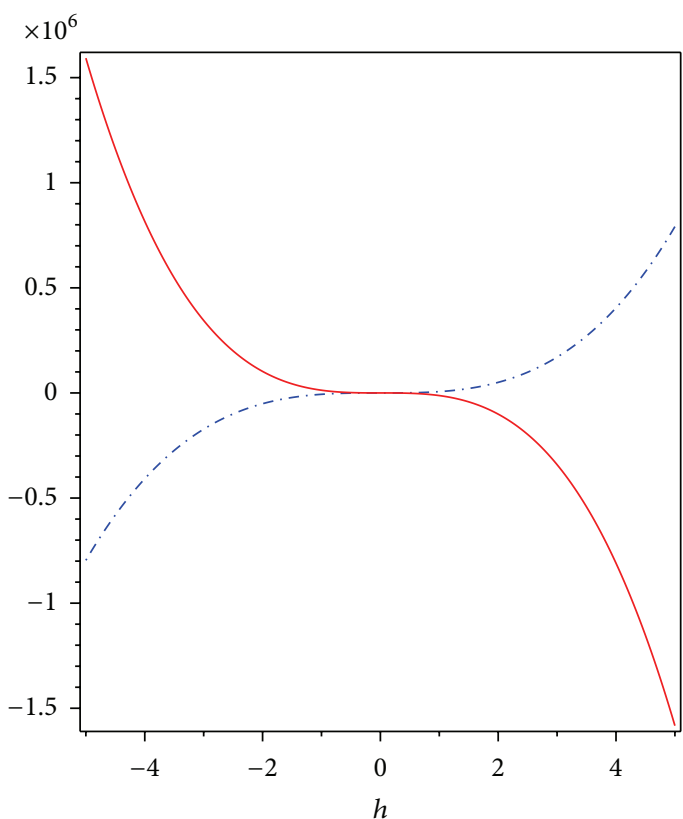

(b)

FIgURE 4: The $h$-curve $y^{\prime \prime}(-1)$ and $y^{\prime \prime \prime}(1)$ for 6th-order (a) SHAM, (b) HAM.

TABLE 4: A comparison of absolute errors between SHAM, LIM, and RHFS.

\begin{tabular}{lccc}
\hline$x$ & SHAM & LIM & $\begin{array}{c}\text { RHFS } \\
k=32\end{array}$ \\
\hline 0.0 & 2nd order $(h=-1)$ & 6 th order & $8.0 \times 10^{-5}$ \\
0.1 & 0 & $1.0 \times 10^{-7}$ & $2.0 \times 10^{-5}$ \\
0.2 & 0 & $7.0 \times 10^{-7}$ & $5.0 \times 10^{-5}$ \\
0.3 & $2.0 \times 10^{-19}$ & $1.0 \times 10^{-6}$ & $1.0 \times 10^{-5}$ \\
0.4 & $1.2 \times 10^{-19}$ & $3.0 \times 10^{-6}$ & $2.0 \times 10^{-5}$ \\
0.5 & 0 & $4.0 \times 10^{-6}$ & $7.0 \times 10^{-5}$ \\
\hline
\end{tabular}

results obtained by the spectral homotopy analysis method, Rationalized Haar functions, and Lagrange interpolation solutions. In Example 1, the numerical results indicate that the rate of convergency in SHAM is faster than HAM. In this example, we found that the forth-order SHAM approximation sufficiently gives a match with the numerical results up to eight decimal places. In contrast, HAM solutions have a good agreement with the numerical results in 20th order with six decimal places. As we can see in Table 4, the spectral homotopy analysis results are more accurate and efficient than Lagrange interpolation solutions and rationalized Haar functions solutions $[27,28]$. As it is shown in Figures 1 and 4 the rang of admissible values of $h$ is much wider in SHAM than HAM.

In this paper, we employed the spectral homotopy analysis method to solve nonlinear Fredholm integro-difflerential equations; however, it remains to be generalized and verified for more complicated integral equations that we consider it as future works.

\section{Acknowledgment}

The authors express their sincere thanks to the referees for the careful and details reading of the earlier version of the paper and very helpful suggestions. The authors also gratefully acknowledge that this research was partially supported by the University Putra Malaysia under the ERGS Grant Scheme having Project no. 5527068.

\section{References}

[1] L. K. Forbes, S. Crozier, and D. M. Doddrell, "Calculating current densities and fields produced by shielded magnetic resonance imaging probes," SIAM Journal on Applied Mathematics, vol. 57, no. 2, pp. 401-425, 1997.

[2] K. Parand, S. Abbasbandy, S. Kazem, and J. A. Rad, "A novel application of radial basis functions for solving a model of firstorder integro-ordinary differential equation," Communications in Nonlinear Science and Numerical Simulation, vol. 16, no. 11, pp. 4250-4258, 2011. 
[3] P. Darania and A. Ebadian, "A method for the numerical solution of the integro-differential equations," Applied Mathematics and Computation, vol. 188, no. 1, pp. 657-668, 2007.

[4] A. Karamete and M. Sezer, "A Taylor collocation method for the solution of linear integro-differential equations," International Journal of Computer Mathematics, vol. 79, no. 9, pp. 987-1000, 2002.

[5] S. J. Liao, The proposed homotopy analysis technique for the solution ofnonlinear problems [Ph.D. thesis], Shanghai Jiao Tong University, Shanghai, China, 1992.

[6] S. J. Liao, The proposed homotopy analysis technique for the solutionof non linear problems [PhD dissertation], Shanghai Jiao Tong University, Shanghai, China, 1992.

[7] S. Liao, Beyond Perturbation. Introduction to the Homotopy Analysis Method, vol. 2 of CRC Series: Modern Mechanics and Mathematics, Chapman \& Hall/CRC, Boca Raton, Fla, USA, 2004.

[8] A. M. Lyapunov, The General Problem of the Stability of Motion, Taylor \& Francis, London, UK, 1992.

[9] G. Adomian, "A review of the decomposition method and some recent results for nonlinear equations," Mathematical and Computer Modelling, vol. 13, no. 7, pp. 17-43, 1990.

[10] G. Adomian and R. Rach, "Noise terms in decomposition solution series," Computers \& Mathematics with Applications, vol. 24, no. 11, pp. 61-64, 1992.

[11] G. Adomian and R. Rach, "Analytic solution of nonlinear boundary value problems in several dimensions by decomposition," Journal of Mathematical Analysis and Applications, vol. 174, no. 1, pp. 118-137, 1993.

[12] G. Adomian, Solving Frontier Problems of Physics: The Decomposition Method, vol. 60 of Fundamental Theories of Physics, Kluwer Academic Publishers, Dordrecht, The Netherlands, 1994.

[13] P. K. Bera and J. Datta, "Linear delta expansion technique for the solution of anharmonic oscillations," PRAMANA Journal of Physics, vol. 68, no. 1, pp. 117-122, 2007.

[14] S. Liao, "On the homotopy analysis method for nonlinear problems," Applied Mathematics and Computation, vol. 147, no. 2, pp. 499-513, 2004.

[15] J. H. He, "The homotopy perturbation method for nonlinear oscillator with discontinuities," Applied Mathematics and Computation, vol. 5, pp. 287-292, 2004.

[16] Sh. S. Behzadi, S. Abbasbandy, T. Allahviranlo, and A. Yildirim, "Application of Homotopy analysis method for solving a class of nonlinear Volterra-Fredholm integro-differential equations," Journal of Applied Analysis and Computation, vol. 1, no. 1, pp. $1-14,2012$.

[17] S. S. Motsa, P. Sibanda, and S. Shateyi, "A new spectralhomotopy analysis method for solving a nonlinear second order BVP," Communications in Nonlinear Science and Numerical Simulation, vol. 15, no. 9, pp. 2293-2302, 2010.

[18] S. S. Motsa, P. Sibanda, F. G. Awad, and S. Shateyi, "A new spectral-homotopy analysis method for the MHD JefferyHamel problem," Computers \& Fluids, vol. 39, no. 7, pp. 12191225, 2010.

[19] S. S. Motsa and P. Sibanda, "A new algorithm for solving singular IVPsof Lane-Emden type," in Proceedings of the 4th International Conferenceon Applied Mathematics, Simulation, Modelling (WSEAS '10), pp. 176-180, Corfu Island, Greece, July 2010.

[20] S. S. Motsa, S. Shateyi, G. T. Marewo, and P. Sibanda, "An improved spectral homotopy analysis method for MHD flow in a semi-porous channel," Numerical Algorithms, vol. 60, no. 3, pp. 463-481, 2012.
[21] H. Saberi Nik, S. Effati, S. S. Motsa, and M. Shirazian, "Spectral homotopy analysismethod and its convergence for solving a class of nonlinear optimalcontrol problems," Numerical Algorithms, 2013.

[22] Z. Pashazadeh Atabakan, A. Kılıçman, and A. Kazemi Nasab, "On spectralhomotopy analysis method for solving Volterra and Fredholm typeof integro-differential equations," Abstract and Applied Analysis, vol. 2012, Article ID 960289, 16 pages, 2012.

[23] W. S. Don and A. Solomonoff, "Accuracy and speed in computing the Chebyshev collocation derivative," SIAM Journal on Scientific Computing, vol. 16, no. 6, pp. 1253-1268, 1995.

[24] A. Molabahrami and F. Khani, "The homotopy analysis method to solve the Burgers-Huxley equation," Nonlinear Analysis. Real World Applications, vol. 10, no. 2, pp. 589-600, 2009.

[25] P. J. Davis and P. Rabinowits, Method of Numerical Integration, Academic Press, London, UK, 2nd edition, 1970.

[26] H. Jafari, M. Alipour, and H. Tajadodi, "Convergence of homotopy perturbation method for solving integral equations," Thai Journal of Mathematics, vol. 8, no. 3, pp. 511-520, 2010.

[27] A. Shahsavaran and A. Shahsavaran, "Application of Lagrange interpolation for nonlinear integro differential equations," Applied Mathematical Sciences, vol. 6, no. 17-20, pp. 887-892, 2012.

[28] F. Mirzaee, "The RHFs for solution of nonlinear Fredholm integro-differential equations," Applied Mathematical Sciences, vol. 5, no. 69-72, pp. 3453-3464, 2011. 


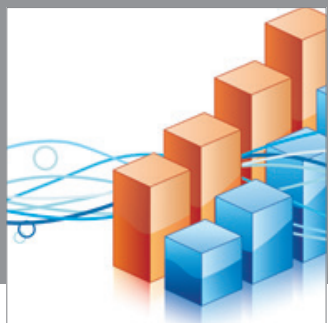

Advances in

Operations Research

mansans

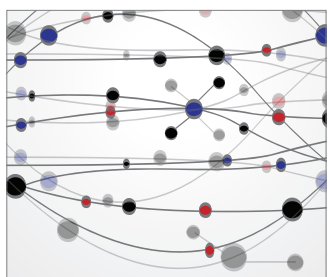

The Scientific World Journal
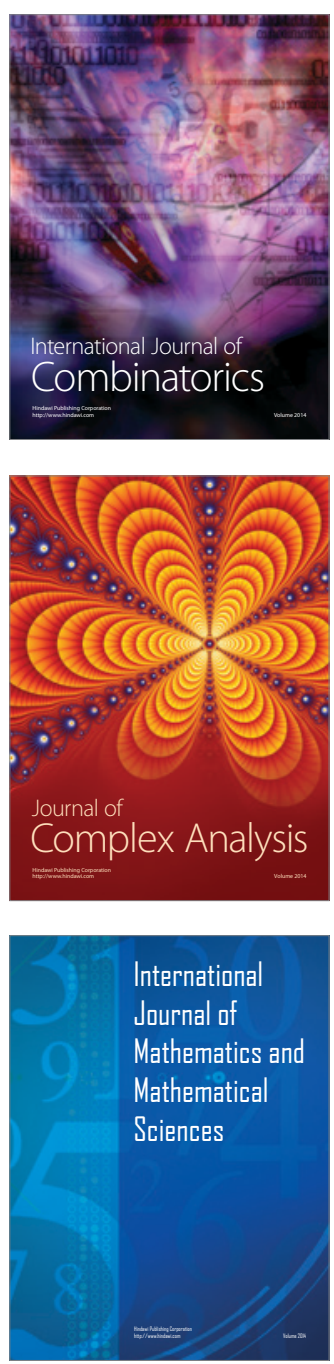
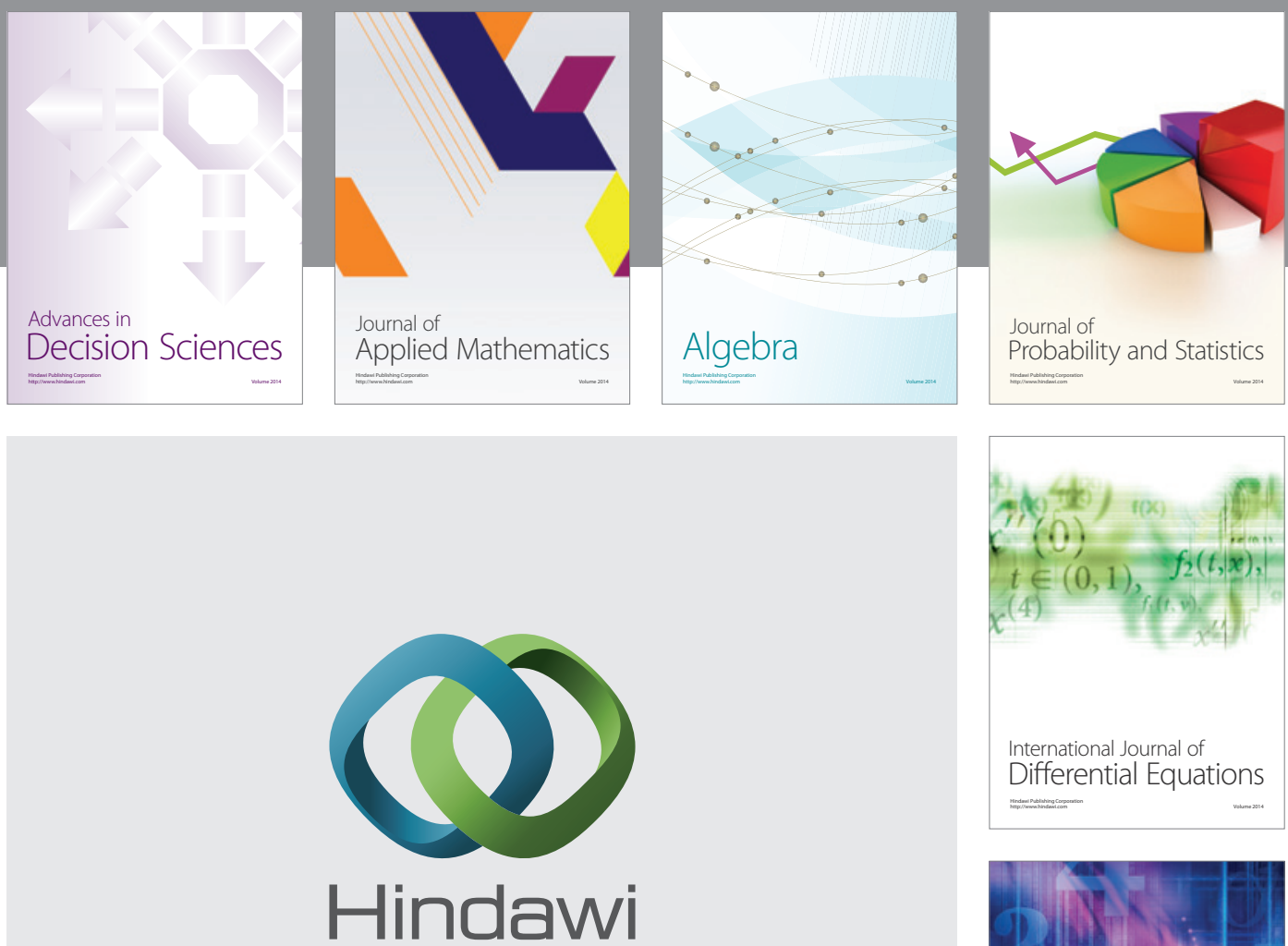

Submit your manuscripts at http://www.hindawi.com
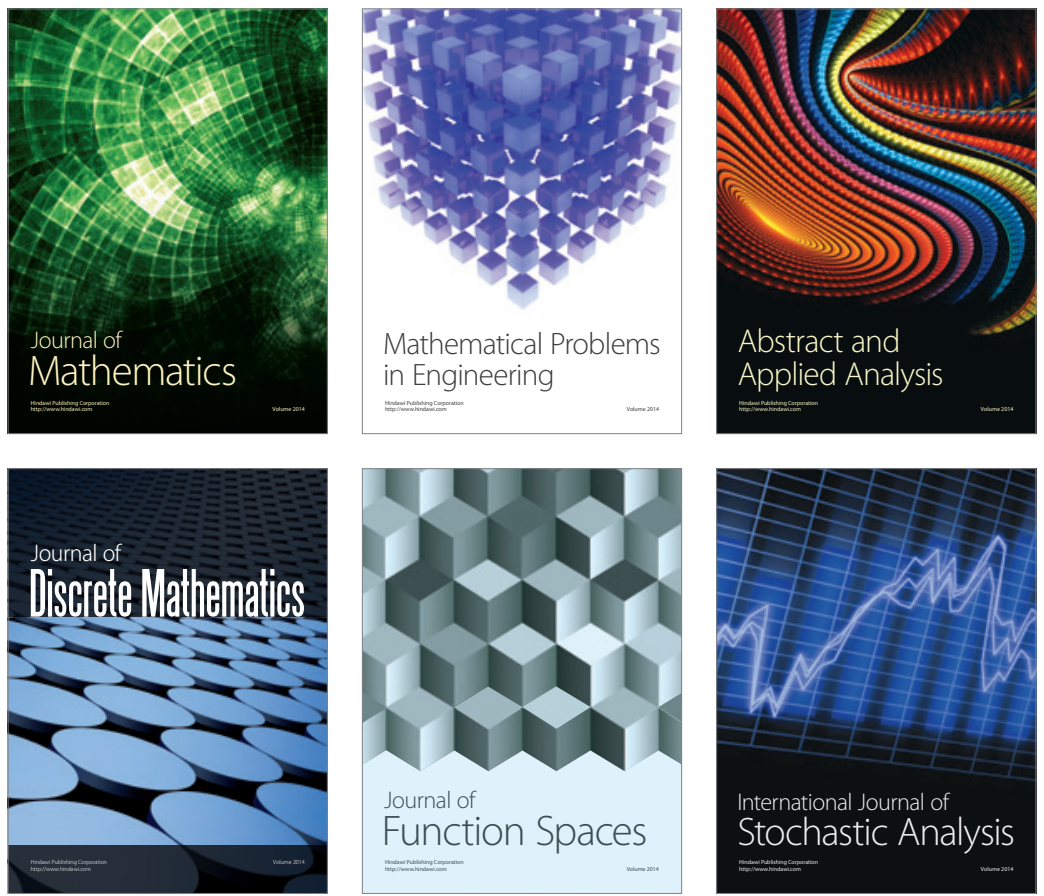

Journal of

Function Spaces

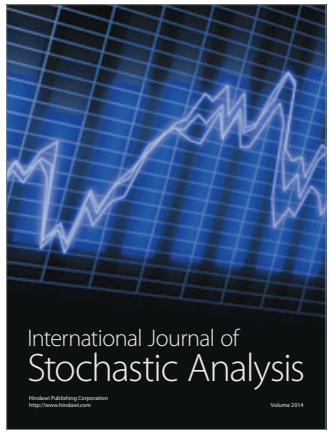

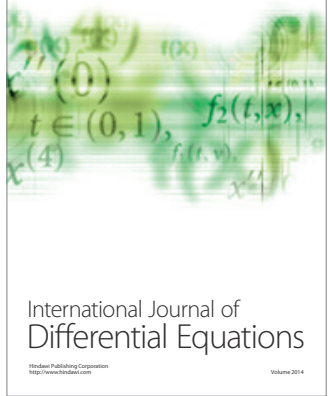
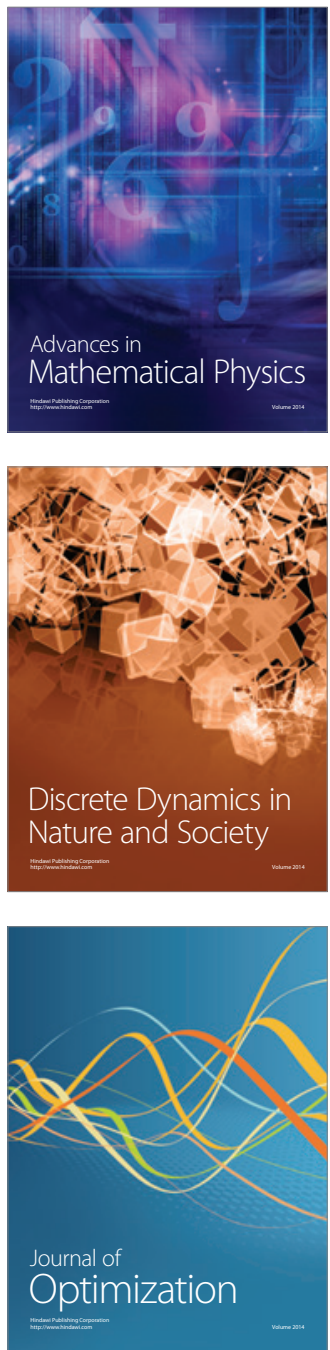